\title{
Development of Tinnitus in CBA/CaJ Mice Following Sound Exposure
}

\author{
Ryan J. Longenecker and Alexander V. Galazyuk \\ Department of Anatomy and Neurobiology, College of Medicine, Northeastern Ohio Universities, 4209 State Route 44, Rootstown, \\ $\mathrm{OH} 44272$, USA
}

Received: 26 February 2011; Accepted: 25 May 2011; Online publication: 11 June 2011

\begin{abstract}
Tinnitus, the perception of a sound without an external acoustic source, is a complex perceptual phenomenon affecting the quality of life in $17 \%$ of the adult population. Despite its ubiquity and morbidity, the pathophysiology of tinnitus is a work in progress, and there is no generally accepted cure or treatment. Development of a reliable common animal model is crucial for tinnitus research and may advance this field. The goal of this study was to develop a tinnitus mouse model. Tinnitus was induced in an experimental group of mice by an exposure to a loud (116 dB sound pressure level (SPL)) narrow band noise (one octave, centered at $16 \mathrm{kHz}$ ) during $1 \mathrm{~h}$ under anesthesia. The tinnitus was then assessed behaviorally by measuring gap induced suppression of the acoustic startle reflex. We found that a vast majority of the sound-exposed mice $(86 \%)$ developed behavioral signs of tinnitus. This was a complex, long lasting, and dynamic process. On the day following exposure, all mice demonstrated signs of acute tinnitus over the entire range of sound frequencies used for testing $(10-31 \mathrm{kHz})$. However, 2-3 months later, a behavioral evidence of tinnitus was evident only at a narrow frequency range $(20-31 \mathrm{kHz})$ representing a presumed chronic condition. Extracellular recordings confirmed a significantly higher rate of spontaneous activity in inferior colliculus neurons in sound-exposed compared to control mice. Surprisingly, unilateral sound exposure suppresses
\end{abstract}

Correspondence to: Alexander V. Galazyuk - Department of Anatomy and Neurobiology - College of Medicine, Northeastern Ohio Universities - 4209 State Route 44, Rootstown, OH 44272, USA. Telephone: (330)325-6640; fax: (330)325-5916; email: agalaz@neoucom.edu startle responses in mice and they remained suppressed even 3 months post-exposure, whereas auditory brainstem response thresholds were completely recovered during 2 months following exposure. In summary, behavioral evidence of tinnitus can be reliably developed in mice by sound exposure, and tinnitus induction can be assessed by quantifying prepulse inhibition of the acoustic startle reflex.

Keywords: Acoustic trauma, startle reflex, spontaneous activity, inferior colliculus, gap-induced suppression of the acoustic startle reflex

\section{INTRODUCTION}

The perceptual phenomenon of tinnitus, commonly described as ringing in the ears, is an affliction that affects nearly one third of Americans (Erlandsson et al. 1992; Ahmad and Seidman 2004). Although much is known about the perceptual aspects of tinnitus, research into its neurophysiological correlates is still in the early stages (Roberts et al. 2010; Engineer et al. 2011). Progress in this field has been reliant on the need for a reliable objective measure of tinnitus.

Over the past two decades, several animal models for tinnitus have been successfully developed (see review by Turner 2007). The vast majority of these models have employed basic mechanisms of conditioning (Bauer et al. 1999; Heffner and Harrington 2002; Guitton et al. 2003; Rüttiger et al. 2003; Lobarinas et al. 2004; Heffner and Koay 2005). They typically require complex behavioral manipulations and weeks to months of animal training. Although these models have contributed significantly to the 
field of tinnitus research, they are time consuming and can be difficult for researchers lacking experience in behavioral techniques. Recently, a novel method was proposed (Turner et al. 2006). It does not require complex behavioral training. Testing can be done quickly in a single 1-h session. This method utilizes reduction in the acoustic startle reflex by a preceding silent gap in an otherwise constant acoustic background. Animals with behavioral evidence of tinnitus cannot detect silence and therefore their reduction of the startle reflex is significantly less than in normal animals. This method has been used successfully to assess tinnitus induced by salicylate overdose or acoustic trauma in rats (Turner et al. 2006; Yang et al. 2007; Kraus et al. 2010).

One strain of mouse, the $\mathrm{CBA} / \mathrm{CaJ}$ strain, is particularly attractive for tinnitus research. These mice have robust hearing capabilities in comparison to other strains and retain normal hearing through most of their life (Davis et al. 2001; Wu and Marcus 2003). The agerelated hearing loss in CBA/CaJ mice is comparable to human hearing models when taking into consideration the differences in life span (Gao et al. 2004). They are also very resilient to noise-induced trauma, which would prevent extreme hearing loss (Davis et al. 2001; Yoshida et al. 2000). Additionally, CBA/CaJ mice have low variability in their responses to noise trauma (Hirose and Liberman 2003), ensuring reliable results during behavioral testing. Perhaps the most important reason for using mice as a tinnitus model is the ability to study knock-in and knock-out mouse models that show behavioral evidence of tinnitus. All these features make mice an ideal model for tinnitus research.

Here, we present results of a study conducted on $\mathrm{CBA} / \mathrm{CaJ}$ mice. Tinnitus was induced with an exposure to a loud narrowband noise. The presence of tinnitus was assessed by measuring the reduction in the acoustic startle reflex by a preceding silent gap in an otherwise constant acoustic background. We found that within a week after exposure, animals showed gap detection deficits at all background frequencies; whereas later, these deficits were predominantly limited to the frequencies higher than the centered frequency of the sound exposure. Our data suggest that a mouse tinnitus model has the capacity to detect and quantify chronic tinnitus caused by sound exposure.

\section{METHODS}

\section{Subjects}

Twenty-two male CBA/CBJ mice were used. Mice were obtained from Jackson Laboratories and were approximately 12 weeks old with a mean weight of $27.5 \mathrm{~g}$ at the beginning of testing. Mice were housed in pairs within a colony room with a 12-h light-dark cycle
(8A.m. to 8 P.M.) at $25^{\circ}$ C. Procedures used in this study were approved by the Institutional Animal Care and Use Committee at the Northeastern Ohio Universities College of Medicine.

\section{Acoustic trauma}

Mice were anesthetized with an intraperitioneal injection of a ketamine/xylazine mixture (100/ $10 \mathrm{mg} / \mathrm{kg}$ ). An additional injection (50\% of the initial dose) was given intramuscularly $30 \mathrm{~min}$ after the initial injection. Mice were exposed to a narrow-band noise centered at $16 \mathrm{kHz}(4-22 \mathrm{kHz})$ unilaterally for $1 \mathrm{~h}$. This noise was generated using a waveform generator (Wavetek model 395), amplified (Sherwood RX-4109) to $116 \mathrm{~dB}$ SPL, and played through a speaker (Fostex FT17H). The outputs of the loudspeaker were calibrated with a 0.25 -in. microphone (Brüel and Kjaer 4135) and found to be $\pm 4 \mathrm{~dB}$ between 10 and $60 \mathrm{kHz}$. A small (2 mm O.D.) plastic tube was used to deliver sound from the speaker to the animal's right ear. The left external ear canal was obstructed with a cotton plug, a manipulation which typically reduces sound levels by at least $30 \mathrm{~dB}$ SPL to a level that does not induce tinnitus (Turner et al. 2006).

\section{Auditory Brainstem Response Testing}

Mice were anesthetized with ketamine/xylazine as in the methods for acoustic trauma. Hearing thresholds were obtained by presenting tone bursts at 10, 16, 20, 24, and $32 \mathrm{kHz}$ at increasing sound intensities ranging from 10 to $80 \mathrm{~dB}$ SPL in $10 \mathrm{~dB}$ steps. Tones were $5 \mathrm{~ms}$ duration, $0.5 \mathrm{~ms}$ rise/fall time and delivered at the rate of $50 / \mathrm{s}$. Auditory brainstem response (ABR) thresholds were obtained before, and immediately after acoustic trauma. Sterile, stainless-steel electrodes were placed subdermally, one behind the right pinna (the ear that was used for noise exposure) and the other along the vertex. The unexposed ear was obstructed with a cotton plug. Evoked potentials were averaged over 300 repetitions. These potentials were amplified (Dagan 2400A preamplifier), filtered (1003,000 $\mathrm{Hz}$ bandpass), digitized (HEKA Elektronik), and stored on a computer hard drive. Thresholds, the smallest sound amplitude that evoked a visible ABR, were determined by visually examining the ABR waveforms in response to every sound frequency presented at different sound levels.

\section{Gap detection testing}

The ability of mice to detect a gap of silence preceding the startle stimulus was determined using commercial hardware/software equipment from 
Kinder Scientific, Inc. Mice were placed in a plastic restrainer situated on a plate with a pressure sensor. Any animal motion was detected by the sensor which measured its amplitude and stored data on the computer hard drive. Kinder Scientific software was used to generate a sequence of stimulus trials including a startle stimulus presented alone (STARTLE) and a startle stimulus paired with a gap (GAP+STARTLE) embedded into continuous background noise; the gap had a $20 \mathrm{~ms}$ duration and $1 \mathrm{~ms}$ rise/fall time (Fig. 1). Background for all these trials was presented as a narrow band ( $1 / 3$ octave) noise centered at six different frequencies $(10,12.5,16,20,25$, and $31.5 \mathrm{kHz})$. This background noise level was constant (75 dB SPL) throughout the session. The startle stimulus was $20 \mathrm{~ms}$ duration white noise presented at $110 \mathrm{~dB}$ SPL, with a $1 \mathrm{~ms}$ rise/fall time. The gap was $20 \mathrm{~ms}$ duration and presented $100 \mathrm{~ms}$ before (onset to onset) the startle stimulus (Fig. 1A). Startle amplitude was measured as the peak-to-peak value (expressed in newtons $(\mathrm{N})$ ) during the 30 -ms time window following startle stimulus onset.

For the gap detection test, parameters of our stimulus paradigm were set to levels which are typical for assuring a robust $\sim 30 \%$ reduction in startle response amplitude caused by a preceding gap of silence in an otherwise continuous background sound (Ison et al. 2002; Turner et al. 2006; Kraus et al 2010).

The testing session started with an acclimation period lasting $3 \mathrm{~min}$. Immediately afterwards, animals received 10 STARTLE-only trials in order to habituate their startle responses to a steady state level. For each of six background frequencies, we presented five

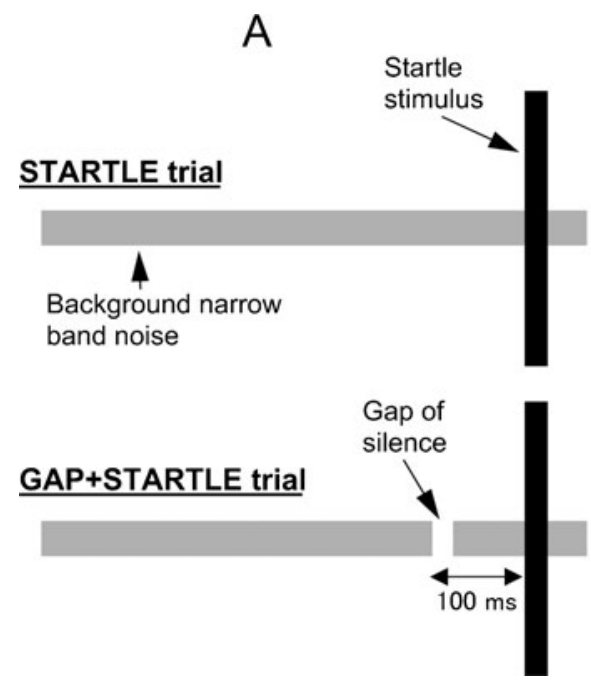

FIG. 1. Two types of stimulus paradigms used for assessing gap detection performance and prepulse inhibition in mice. A Gap detection stimulus paradigm consists of (1) STARTLE trial-a startle stimulus of wide band noise ( $20 \mathrm{~ms}$ duration, $110 \mathrm{~dB} \mathrm{SPL}$ ) embedded in a continuous background narrow band noise centered at 10, 12.5, 16, 20, 25, and $31.5 \mathrm{kHz}$ and presented at $75 \mathrm{~dB} \mathrm{SPL}$; (2) GAP STARTLE trial-similar to the STARTLE trial with the addition of a
STARTLE only trials and five GAP+STARTLE trials. The STARTLE and GAP+STARTLE trials were pseudo-randomized. The inter-trial intervals were also pseudo-randomized between 7 and $15 \mathrm{~s}$. After we completed testing all six background frequencies, the entire session was repeated one more time. Thus, during this testing for each background frequency, the total of 10 GAP+STARTLE trials and 10 STARTLE only trials were presented.

Every animal from the experimental group was tested before acoustic trauma, and then at several time points afterward: $1,3-5,7$ days, weekly for 2 months, and 3 months post-exposure. The control group of mice was tested at the same time points.

\section{Prepulse detection testing}

The prepulse session contained two types of stimuli (Fig. 1B). First, a startle stimulus was presented in silence and had the same parameters as the startle during the gap detection session. The second stimulus type was the startle stimulus preceded by a prepulse. The prepulse stimuli were $20 \mathrm{~ms}$ duration with a $1 \mathrm{~ms}$ rise/fall time and presented at the same six different narrow band noise frequencies as in the gap detection session. For each frequency, the mice were given five startle stimulus-alone trials, intermingled with five trials containing a prepulse (Fig. 1B).

\section{Data analysis}

Startle responses showed some variability during the recording sessions: some animals sometimes exhibited

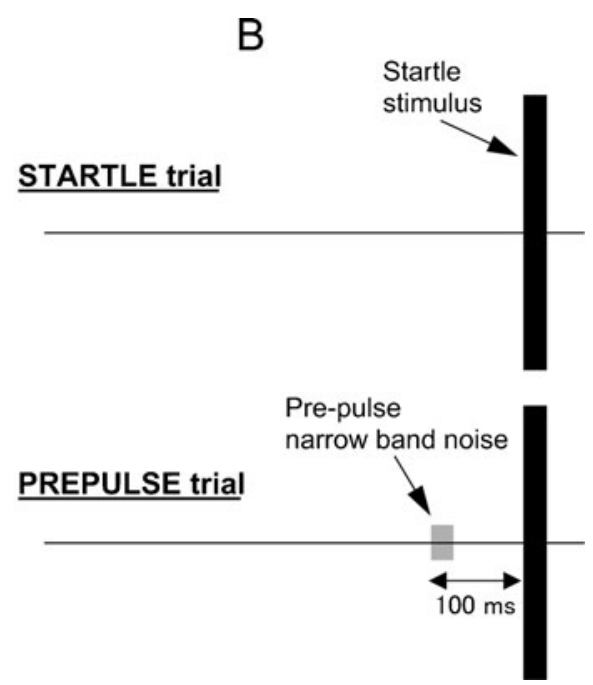

$20 \mathrm{~ms}$ gap of silence embedded $100 \mathrm{~ms}$ before the startle stimulus. B The prepulse detection stimulus paradigm contains (1) STARTLE trial -a startle stimulus (the same as in A) presented in silence; (2) PREPULSE trial - a startle stimulus was preceded by a prepulse narrow band noise (20 ms duration, $75 \mathrm{~dB} \mathrm{SPL}$ ) centered at six different frequencies (the same as in the gap detection paradigm). 
an extremely strong startle response or did not startle at all. Therefore, the data in each session were trimmed by eliminating the highest and the lowest startle responses for each frequency (two of 10 responses). A suppression ratio was then calculated by dividing the amplitude of the startle response recorded during GAP+STARTLE trials by the STARTLE only trial (Fig. 1). These ratios are shown on the majority of figures in this paper. One-way analyses of variance were used for statistical analysis. The criterion for the presence of behavioral evidence of tinnitus was a significant reduction in gap detection performance at one or several background frequencies compared to the pre-exposure values. During our data analysis, we found empirically that one standard error confidence limit is an optimal must-reach criterion to demonstrate changes in gap or prepulse detection performance induced by sound exposure.

While significant changes in gap or prepulse detection performance could theoretically be increased or decreased, only significant increases were observed in our study.

\section{Extracellular recording}

Two mice from the control and two from the sound exposed groups were used for extracellular recordings. Each mouse was anesthetized using isoflurane inhalation $(1.5-2.0 \%$, isoflurane administered by a precision vaporizer) prior to surgery. A midline incision of the skin over the cranium was made. The tissue overlying the skull then was removed and a small metal rod was glued to the skull using glass ionomer cement (3 M ESPE, Germany). Following surgery, animals were allowed to recover for 1-2 days in individual holding cages.

Two days after surgery, each mouse was trained to stay inside a small plastic tube, to be used as a holding device during recording sessions. The metal rod on the head of the mouse was secured to a small holder designed to restrain the head of the animal without causing distress, while the ears were unobstructed for free-field acoustic stimulation. Recordings were made from the contralateral inferior colluculus (IC) in awake mice inside a single-walled sound attenuating chamber (Industrial Acoustics Company, Inc). Throughout the recording session $(3-4 \mathrm{~h})$, the animal was offered water periodically and monitored for signs of discomfort. After a recording session, the exposed skull was covered with sterile bone wax and the animal was returned to its holding cage. Experiments were conducted every $2-3$ days for a maximum of 2 weeks. No sedative drugs were used during recording sessions. If the animal showed any signs of discomfort, the recording session was terminated and the mouse was returned to its cage.
A small hole $(\sim 50 \mu \mathrm{m})$ penetrating the dura was drilled in the skull overlying the IC, through which a recording electrode was inserted into the IC. Extracellular single-unit recordings were made with borosilicate glass micropipettes (10-20 M $\Omega$ impedance, 2$3 \mu \mathrm{m}$ tip) filled with $0.5 \mathrm{M}$ sodium acetate. The electrode was positioned into the drilled hole by means of a precision $(1 \mu \mathrm{m})$ digital micromanipulator using a surgical microscope (Leica MZ9.5). The relative position of each electrode was monitored from the readouts of digital micrometers using a common reference point on the skull. Vertical advancement of the electrode was made by a precision piezoelectric microdrive (Model 660, KOPF Instr.) from outside the sound-attenuating chamber. Recorded action potentials were amplified (Dagan 2400A preamplifier), monitored audiovisually on a digital oscilloscope (DL1640, YOKOGAWA), digitized and then stored on a computer hard drive using EPC10 digital interface and PULSE software from HEKA Elektronik at a bandwidth of $100 \mathrm{kHz}$.

\section{RESULTS}

For this study, $22 \mathrm{CBA} / \mathrm{CaJ}$ mice were divided into two groups: experimental $(n=14)$ and control $(n=8)$. The experimental mice were exposed to a loud $(116 \mathrm{~dB}$ SPL) narrowband (one octave) noise centered at $16 \mathrm{kHz}$ unilaterally for $1 \mathrm{~h}$ under general anesthesia. The control mice were simply anesthetized for $1 \mathrm{~h}$.

The control and sound-exposed mice were behaviorally tested before exposure and from days 1 to 84 post-exposure. For both groups, we measured the GAP+STARTLE/STARTLE $((G+S) / S)$ and PREPULSE+STARTLE/STARTLE $((P+S) / S)$ ratios by dividing the amplitude of startle responses preceded by either a gap or by a prepulse by the amplitude of startle stimuli presented alone. During gap testing, a continuous background narrow-band noise centered at six different frequencies $(10,12.5,16,20,25$, and $31.5 \mathrm{kHz}$ ) was presented at $75 \mathrm{~dB}$ SPL (Fig. 1A). During prepulse detection testing, the prepulse and a startle stimuli were presented in silence. For both these tests, a ratio of 1 means that the animal does not detect a gap or a prepulse; whereas, ratio values lower than 1 indicates better detection.

\section{GAP detection performance in the control mice}

We found that all control mice without exception showed a robust suppression of their startle response when a GAP was introduced. The $(G+S) / S$ ratio values computed as an averaged ratio over all six background frequencies varied among mice from approximately $0.3-0.75 \quad($ mean $\pm \mathrm{SD}=0.617 \pm 0.14)$ yet 
did not change significantly within each mouse during 3 months of testing. Such ratios also varied from one background frequency to another in individual mice. For a representative mouse in Figure 2, this ratio ranged from 0.4 to $0.86($ mean $\pm \mathrm{SD}=0.66 \pm 0.1)$ for six different frequencies. As shown in Figure 2, the overall fluctuations of $(G+S) / S$ ratios were not significantly different $(F(4,50)=0.14, p=0.966)$ when
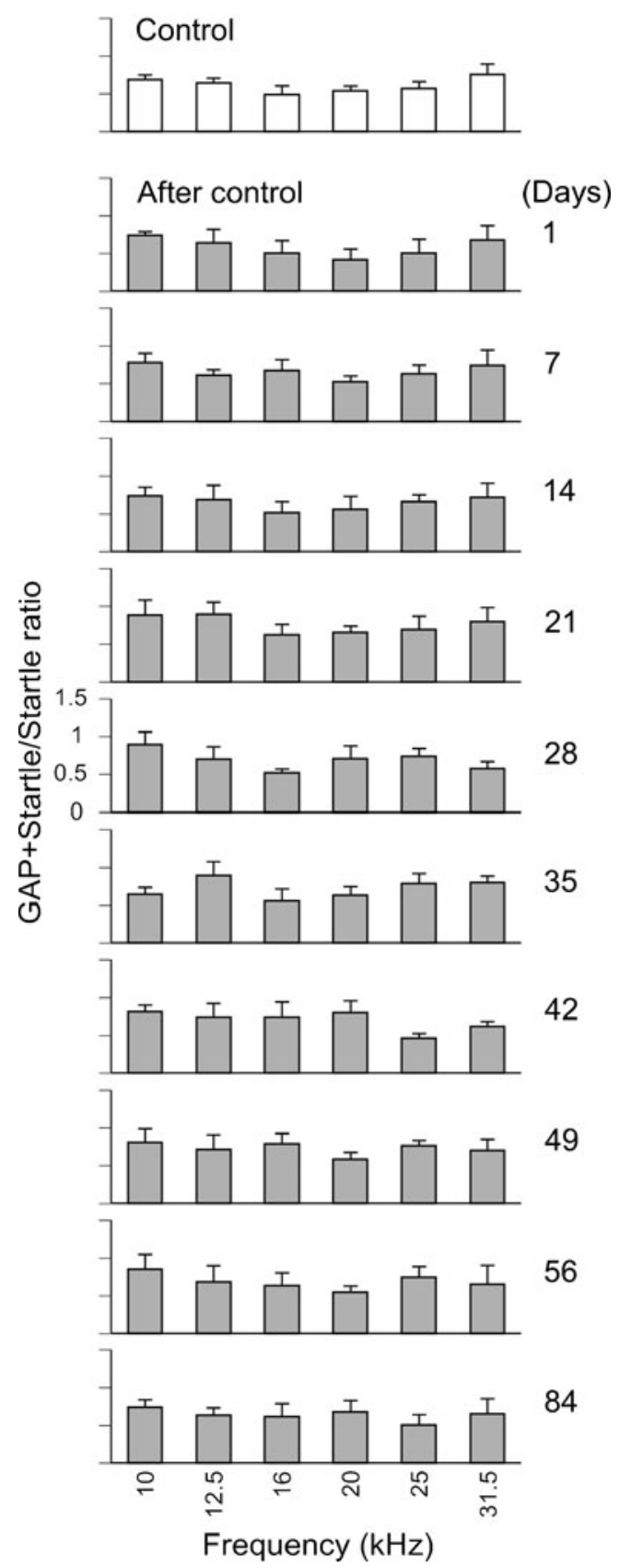

FIG. 2. Fluctuations of gap-induced suppression of the acoustic startle response over a 3-month period in a mouse from the control group. Open bars represent mean \pm SEM of $(G+S) / S$ ratios measured at six different background frequencies $(10,12.5,16,20,25$, and $31.5 \mathrm{kHz}$ ) and serve as a control. The gray bars represent ratios measured at the same frequencies but at different time points after control measurements. measured at different time points. Consistent with other studies, our control group of mice exhibit robust gap-induced inhibition of the startle response (Turner et al. 2006; Yang et al. 2007; Wang et al. 2009; Kraus et al. 2010).

\section{GAP detection performance in the sound exposed mice}

In the experimental mice before the sound exposure, the gap detection performance $((G+S) / S$ mean $\pm \mathrm{SD}=$ $0.608 \pm 0.21)$ was not significantly different from the control group of mice $(F(1,200)=0.76, p=0.384)$. However, after unilateral sound exposure, the values of $(G+S) / S$ ratios for all exposed mice became much higher (mean $\pm \mathrm{SD}=0.94 \pm 0.12$ ), indicating that the gap detection was significantly reduced $(F(1,200)=$ $37.19, p<0.0001)$. A representative mouse in Figure 3A before sound exposure showed good gap detection performance with $(G+S) / S$ ratios ranging from 0.25 at $25 \mathrm{kHz}$ to 0.61 at $10 \mathrm{kHz}($ mean $\pm \mathrm{SD}=$ $0.45 \pm 0.12$; Fig. 3A, top panel). However, on the first day after exposure, this gap detection performance was significantly decreased at all background frequencies. The averaged $(G+S) / S$ ratio across all six frequencies was increased (mean $\pm \mathrm{SD}=0.86 \pm 0.09$ ). Until day 21 following sound exposure, this mouse still showed low gap detection performance at 5/6 different frequencies. Beginning at day 28 after exposure, gap detection started to return to the control level at more and more background frequencies. From days 42 to 56, the gap detection deficits were evident only at one or two background frequencies between 16 and $20 \mathrm{kHz}$. From days 56 to 84, these deficits were shifted to higher frequencies (Fig. 3A, bottom panel). At this time period after exposure, the gap detection performance was significantly lower at 20 and $25 \mathrm{kHz}$. Similarly, the vast majority of the exposed mice $(86 \%, 12 / 14)$ developed behavioral signs of tinnitus. Figure $3 \mathrm{~B}$ shows a distribution of significant increases in the ratios as a function of background frequency (e.g., indicated by black bars in Fig. 3A) obtained from a population of 12 soundexposed mice. By day 84 after exposure, gap detection deficits were predominantly found between 20 and $31.5 \mathrm{kHz}$. The range of frequencies with evidence of tinnitus in addition to $20 \mathrm{kHz}$ was similar to that in the mice shown in Fig. 3A. Four of 12 mice showed gap detection deficits at one additional (higher or lower) frequency, 5/12 mice showed deficits at two additional frequencies, and the three remaining mice exhibited such deficits at three additional frequencies. Our data suggest that the development of behavioral signs of tinnitus after high-level noise exposure in mice is a complex, long lasting, and dynamic process. 


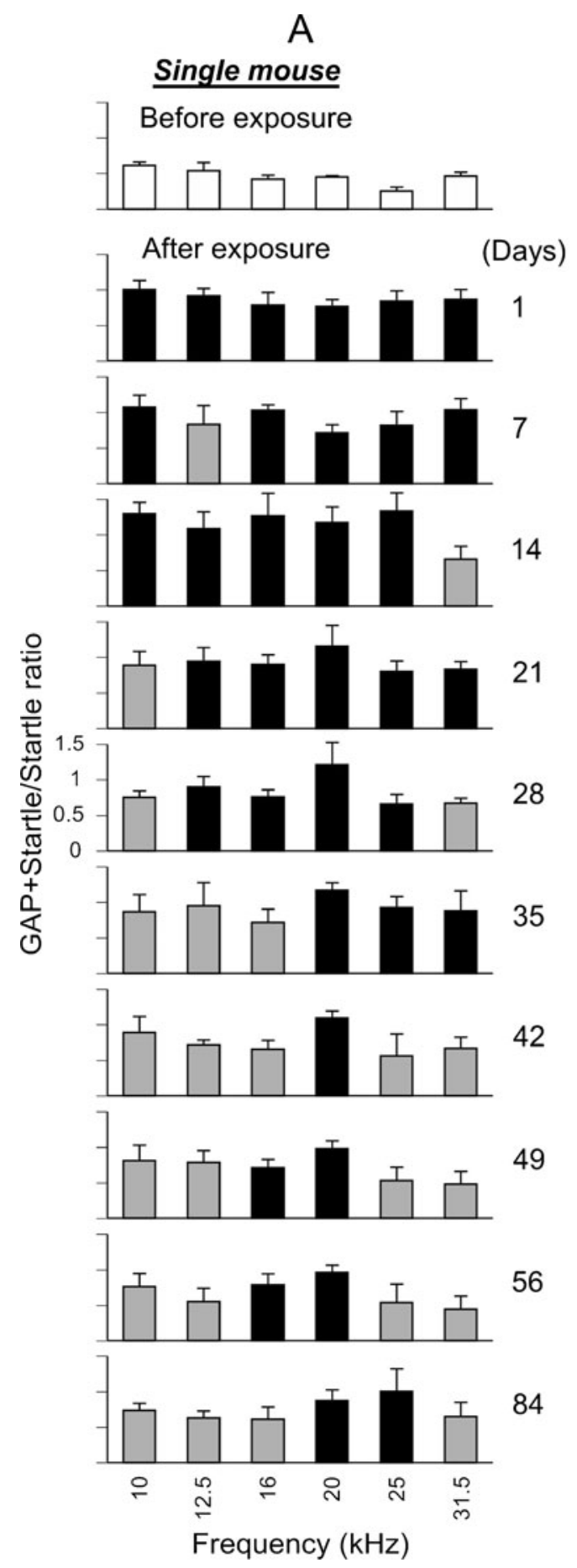

FIG. 3. Time-dependent changes of gap-induced suppression of the acoustic startle response in mice during 3 months after sound exposure. A Changes in gap detection performance in a single soundexposed mouse. Open bars represent mean \pm SEM of $(G+S) / S$ ratios measured before sound exposure. Gray and black bars represent the ratios, which were not (gray bars) or were (black bars) significantly different from the control. B A histogram depicts only significant increases in the ratios as a function of background frequency (e.g., indicated by black bars in A) obtained from a population of 12 sound-exposed mice.

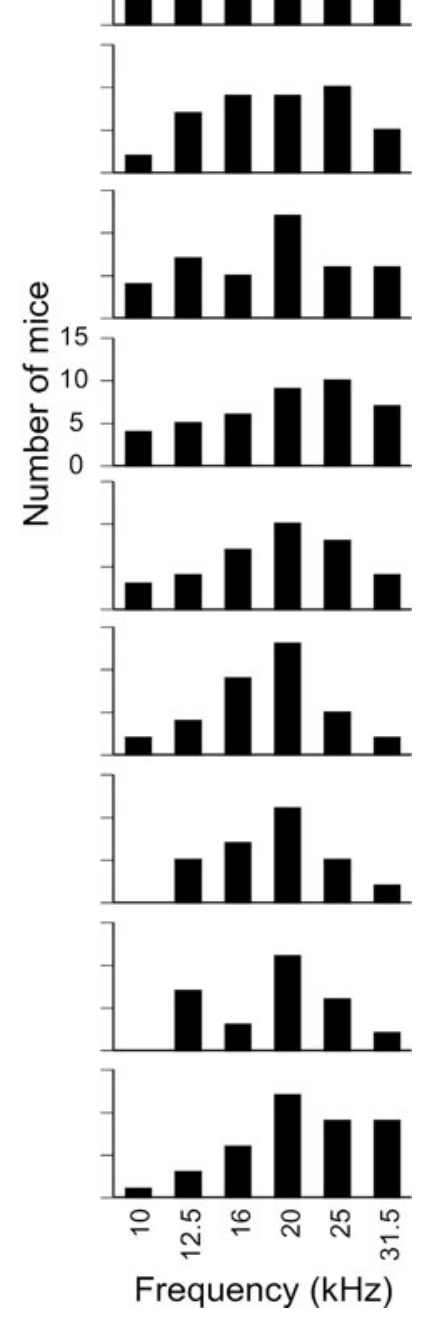

Prepulse detection performance in the control and sound-exposed mice

All control and sound-exposed mice were tested for prepulse inhibition of the acoustic startle response. Basically, this test was the inverse of the gap detection test. Instead of a gap of silence embedded into continuous background narrow-band noise, a prepulse was presented before the startle in silence. This prepulse had the same duration and amplitude as a gap. The narrow band noises for the prepulse were centered at the same six frequencies $(10,12.5,16,20$, 25 , and $31.5 \mathrm{kHz}$ ) as the background noise during the gap detection test.

Prepulse detection testing served as a control for possible hearing loss and temporal deficits that might explain gap detection deficits. A loss in gap detection performance accompanied by deficits in prepulse detection would suggest hearing loss or a temporal processing dysfunction as the main reason of the gap 
deficit. However, deficits in gap detection not accompanied by deficits in prepulse detection suggest the presence of tinnitus.

The control group of mice showed a robust prepulse inhibition. The $((P+S) / S)$ ratio values computed as an averaged ratio over all six background frequencies varied among mice from approximately $0.1-0.47($ mean $\pm \mathrm{SD}=0.236 \pm 0.07)$ yet did not change significantly for each mouse during 3 months of testing. A representative mouse from this population is shown in Figure 4. This mouse exhibited ratios ranging from 0.11 to 0.41 (mean $\pm \mathrm{SD}=0.24 \pm 0.08$ ) for six different frequencies. The fluctuations of the ratio were not significantly different $(F(4,25)=0.83, p=$ 0.519 ) when measured at different time points.

In the sound-exposed mice before the sound exposure, the average of ratios (mean $\pm \mathrm{SD}=0.24 \pm$ 0.11 ) was not significantly different from the control group of mice $(F(1,70)=0.05, p=0.824)$. However, sound exposure altered these ratios in the vast majority of these mice (12/14). The changes in the ratios after exposure were somewhat different from those for gap detection. In contrast to gap testing, a majority of sound exposed mice (8/12) showed only modest change on the first day post-exposure. Furthermore, three of 12 mice exhibited an increase in prepulse inhibition because their ratios were slightly decreased. A representative mouse in Figure 5A showed no changes in ratios on the first day after exposure. However, by day 3 , the ratios for $10,12.5$, 20 , and $25 \mathrm{kHz}$ were significantly higher than that for the control. Between days 3 and 35 post-exposure, the ratios were similar to the control except in single frequencies at days 21 and 28. Interestingly, later between days 42 and 49 post-exposure, the ratios were elevated again. At days 56 and 84 post-exposure, $((P+$ $S) / S$ ) ratios for the vast majority of tested frequencies were not different from the control. The population data from 12 mice showed a similar pattern of timedependent changes in significantly elevated ratios after sound exposure (Fig. 5B). By day 84 postexposure all but three mice exhibited complete recovery of prepulse detection performance to the control level. In three out of 12 mice, small deficits in prepulse detection were still evident in the range of 20-31.5 kHz.

\section{Effect of sound exposure on ABR}

The thresholds of ABR were measured in the soundexposed groups of mice $(N=12)$ before, immediately after, 2, and 3 months following exposure at five different frequencies $(10,16,20,24$, and $32 \mathrm{kHz}$; Fig. 6). Immediately following sound exposure, all mice showed significantly elevated thresholds for all five frequencies $(F(1,175)=755.85, p<0.0001$, Fig. $6 \mathrm{~A})$.
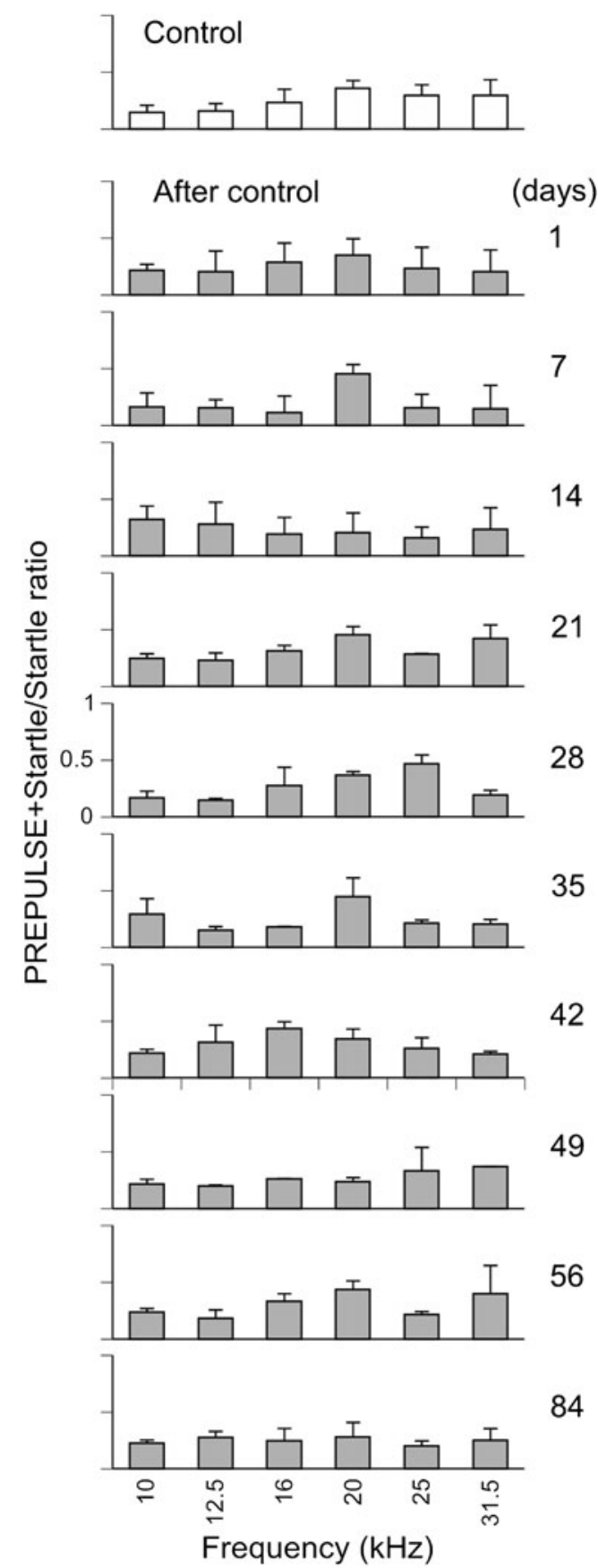

FIG. 4. Fluctuations of prepulse detection performance over a 3month period in a mouse from the control group. Open bars represent mean \pm SEM of ratios measured at six different background frequencies $(10,12.5,16,20,25$, and $31.5 \mathrm{kHz})$ and serve as a control. The gray bars represent ratios measured at different time points after control measurements.

Fifty six days post-exposure, a significant threshold shift was evident at $24 \mathrm{kHz}$ only (Fig. 6B). Although not significant, thresholds for the remaining four frequencies were slightly elevated relative to the pre-exposure level. By day 84 post-exposure, the ABR thresholds for all five frequencies were completely recovered and were not significantly differ- 


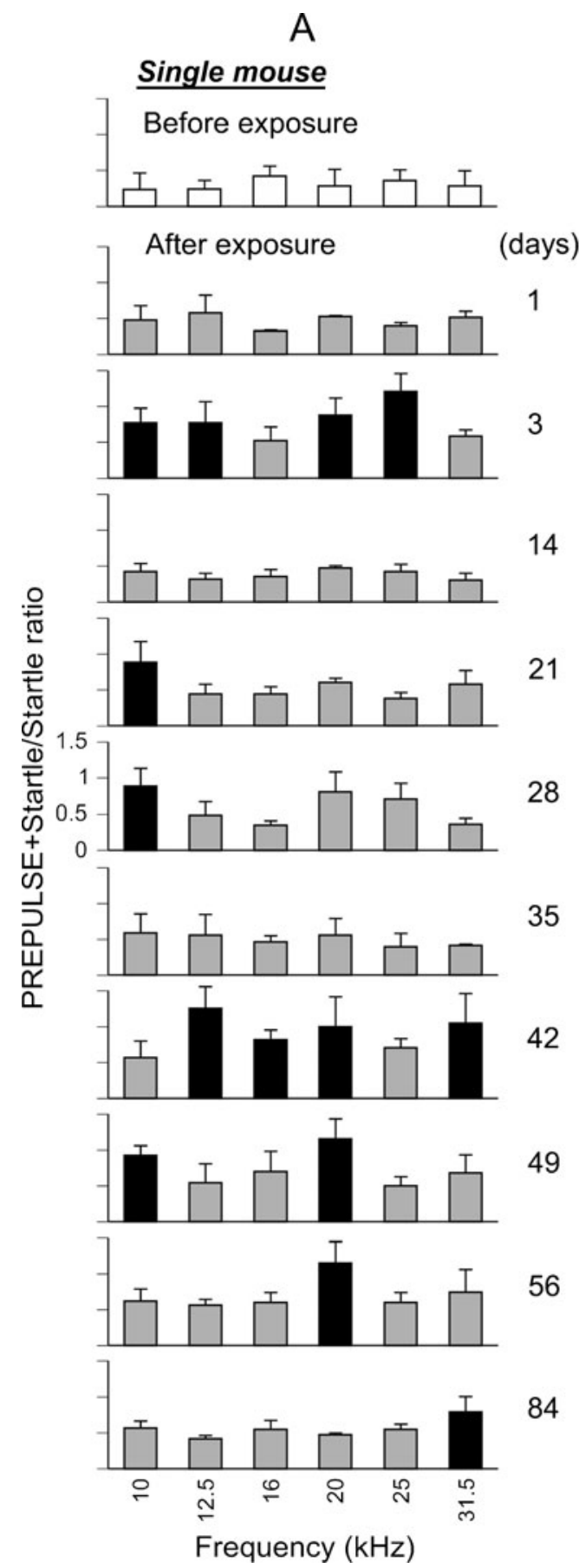

FIG. 5. Time-dependent changes of prepulse induced suppression of the acoustic startle response in mice during 3 months after sound exposure. A Changes of prepulse detection performance in a single mouse. Open bars represent mean \pm SEM of ratios measured before sound exposure. Gray and black bars represent the ratios, which were not (gray bars) or were (black bars) significantly different from the control. B A histogram depicts only significant increases in the ratios as a function of background frequency obtained from a population of 12 soundexposed mice.

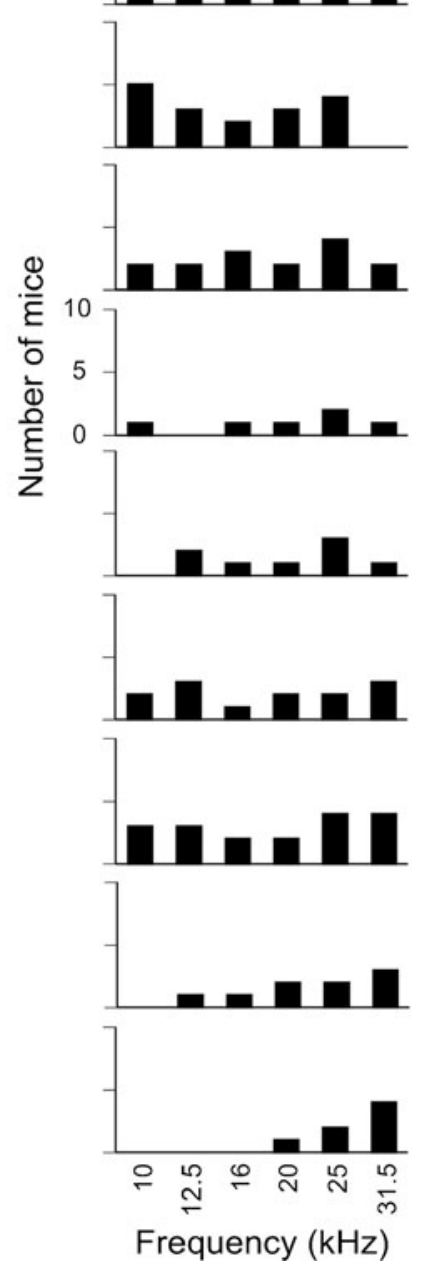

ent from the control level $(F(1,143)=0.2, p=0.6554$; Fig. 6C).

\section{Effect of sound exposure on startle amplitude}

The absolute value of the amplitude of the startle response may be affected by sound exposure. To test for this possibility, the absolute amplitude of startle responses was measured for both the control and sound exposed mice from days 1 to 84 post-exposure.
The amplitude of the startle response in the control group of mice varied among animals and ranged from 0.09 to $0.36 \mathrm{~N}$ (mean $\pm \mathrm{SD}=0.22 \pm 0.06$; Fig. $7 \mathrm{~A})$. The average value from eight mice in the control group did not change significantly when measured during the 3-month period $(F(4,35)=0.52$, $p=0.722$ ). The experimental group of mice also showed very similar averaged values of the startle response before sound exposure. Their startle amplitude ranged from 0.07 to $0.34 \mathrm{~N}($ mean $\pm \mathrm{SD}=0.23 \pm$ 


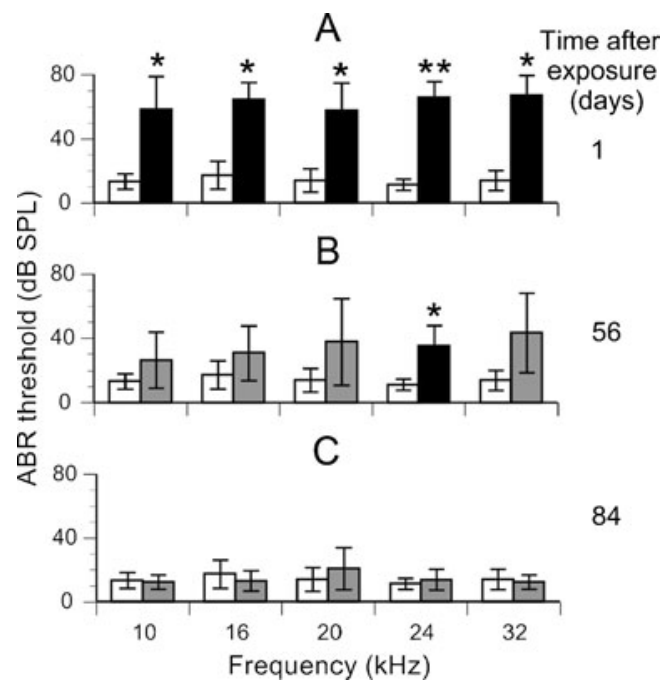

FIG. 6. Effects of sound exposure on ABR thresholds for 12 experimental mice. A Mean ABR thresholds at five frequencies (10, $16,20,24$, and $32 \mathrm{kHz}$ ) recorded before (open bars) and immediately after sound exposure (black bars). B-C Mean ABR thresholds recorded 56 and 84 days after exposure, respectively. Black and gray bars were and were not, respectively, significantly different from presound exposure responses; ${ }^{*} p<0.05,{ }^{* *} p<0.001$.

0.08). However, on the first day after exposure, these values were greatly reduced (mean $\pm \mathrm{SD}=0.08 \pm 0.03$; Fig. 7B). From days 1 to 84 following sound exposure, the startle amplitude slightly increased and ranged from 0.03 to $0.27 \mathrm{~N}($ mean $\pm \mathrm{SD}=0.11 \pm 0.04)$ and remained significantly lower than the pre-exposure level $(F(1,103)=114.32, p<0.0001)$.

The reduction in startle amplitude after sound exposure raised a concern that the startle amplitude was too low to be inhibited by a preceding gap or a prepulse. To address this issue, we compared the suppressed startle amplitudes in 12 sound-exposed mice with the amplitudes of baseline movements measured during the trials when neither startle stimulus nor background narrow band noise were presented. We found that even suppressed startle amplitudes were significantly higher than baseline movements $(F(1,146)=284.5, p<0.0001)$ suggesting that this amplitude was still high enough to be inhibited by a preceding gap or by a prepulse.

\section{Effect of sound exposure on spontaneous activity} in the IC

Spontaneous activity of IC neurons was recorded in two control mice and two sound-exposed mice 5 weeks post-exposure (Fig. 8). The vast majority of the IC neurons in unexposed mice $(96 \%, 74 / 77)$ fired spontaneously. The firing rates for these neurons ranged from 0.2 to 35 spikes per second $(\mathrm{sp} / \mathrm{s})$ with a mean of $5.94 \mathrm{sp} / \mathrm{s}$. The mean value was skewed by very high spontaneous rates in a few neurons; hence, the median spontaneous firing rate of $2.5 \mathrm{sp} / \mathrm{s}$ better reflects the population (Fig. 8A).

The sound-exposed mice showed higher rates of spontaneous activity in the IC relative to the unexposed mice (Mann-Whitney, $U=931.5, p<0.0001$, twotailed; Fig. 8B). The firing rates of IC neurons in exposed mice ranged from 0.5 to $95 \mathrm{sp} / \mathrm{s}$, with a mean of $25.94 \mathrm{sp} / \mathrm{s}$ and it was five times higher than that of controls.

\section{DISCUSSION}

This study is the first application of the recently developed technique utilizing gap-induced suppression of acoustic startle reflex to assess tinnitus in the
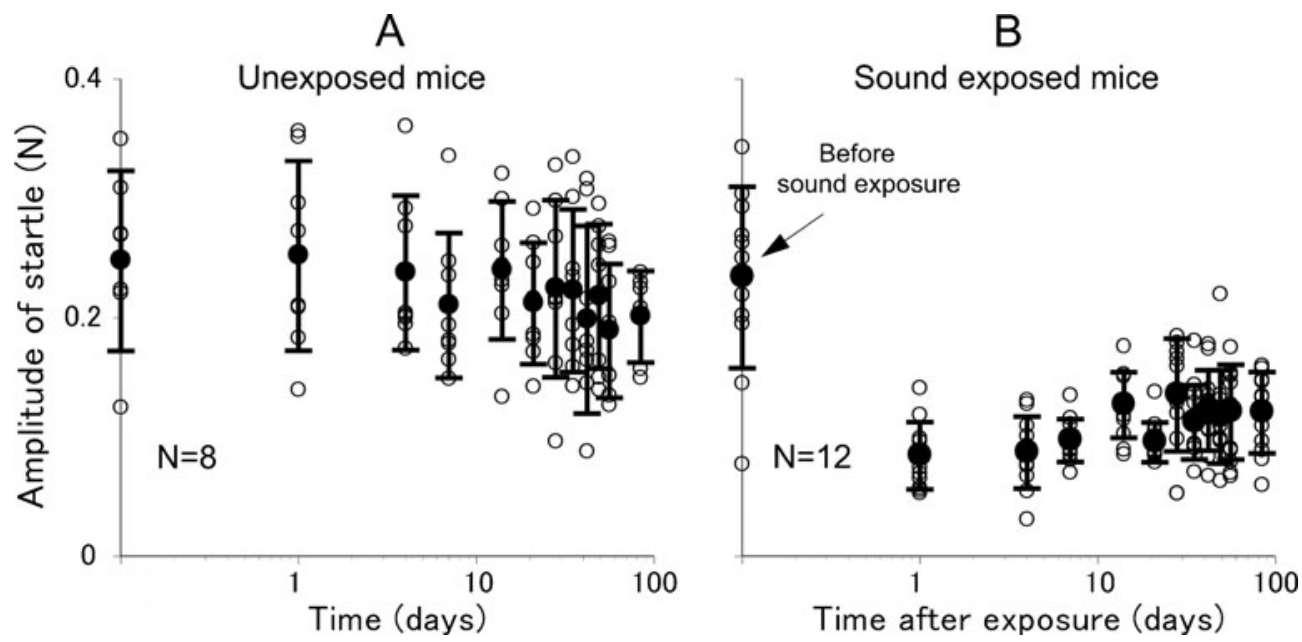

FIG. 7. Reduction in acoustic startle response amplitude after sound exposure. A Individual data points (open circles) and mean \pm SEM values from eight control mice recorded over a 3-month period. B The same data collected from 12 experimental mice before and at different times after sound exposure. 

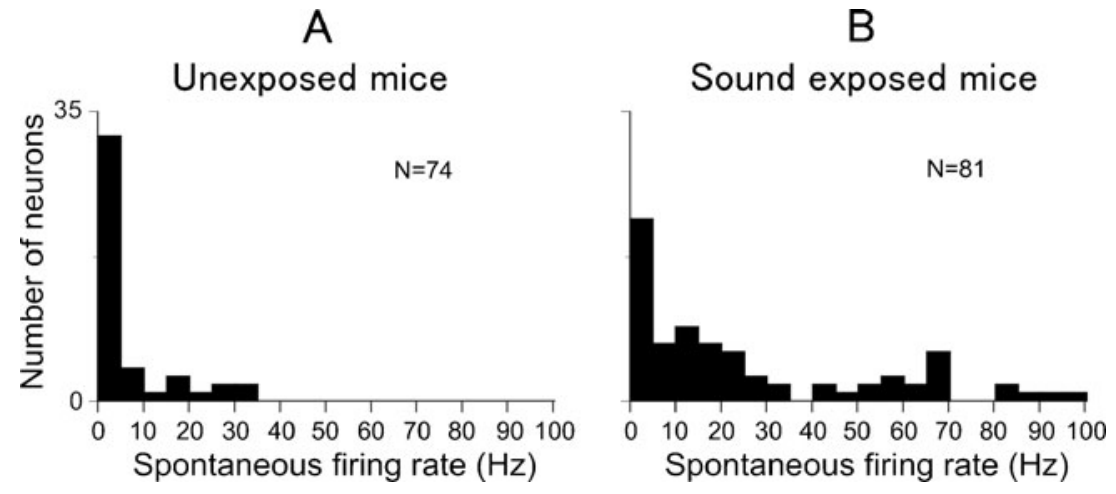

FIG. 8. Increase in spontaneous firing rate of IC neurons following sound exposure. A-B Distribution of IC neurons exhibiting different spontaneous firing rates in control (unexposed) and experimental (sound-exposed) animals, respectively, 2 months after sound exposure. Recordings were conducted in the IC contralateral to the exposed ear. mouse model (Turner et al. 2006). Our research produced four novel, significant observations. First, we demonstrated that mice can be successfully used as an animal model of tinnitus. Second, we described distinct time courses of changes in gap and prepulse detecting performance. Third, we showed that unilateral sound exposure suppresses startle responses and they remain suppressed 3 months post-exposure. Lastly, we confirmed that inferior colliculus neurons of the mice with behavioral evidence of tinnitus exhibit abnormally high spontaneous firing rates.

We found that in a CBA/CaJ strain of mice, sound exposure triggered a chain of transformations in the auditory system including gap and prepulse detection performances, ABR amplitude, and startle response amplitude. These transformations exhibited a distinct pattern of change: right after sound exposure, behavioral signs of tinnitus were evident at a wide range of sound frequencies; yet by the third month postexposure, these changes occurred predominantly within a narrow frequency range. In agreement with another study performed on rats, this range was shifted to higher frequencies outside the frequency range of sound-induced acoustic trauma (Wang et al. 2009). In agreement with other studies (Salvi et al. 1996; Kaltenbach and Afman 2000; Brozoski et al. 2002; Mulders and Robertson 2009; Dong et al. 2010; Mulders et al. 2010), we also found that behavioral signs of tinnitus were accompanied by an elevation of spontaneous activity in inferior colliculus neurons. Although, our electrophysiological results confirm that sound exposure leads to hyperactivity, it does not explain the origin of this hyperactivity. Whether this hyperactivity in the inferior colliculusis is inherited from the brainstem (in brainstem: Kaltenbach and Afman 2000; Kaltenbach et al. 2000; in inferior colliculus: Mulders and Robertson 2009) would seem relevant and is worth further investigation. To clarify whether hyperactivity is always linked to tinnitus or it is present only during tinnitus development, we have decided to monitor our sound-exposed population of mice for one more year. We hope that such data might shed light on these issues.
The gap detection results indicate that by 3 months post-exposure, the mice developed deficits in gap detection performance predominantly at the frequency range of $20-31.5 \mathrm{kHz}$. This deficiency could plausibly be due to hearing loss in this frequency range. Alternatively, it could be explained by the presence of behavioral signs of tinnitus, which would preclude an animal from detecting a gap of silence in a continuous background. Several pieces of evidence suggest that hearing loss is not a likely explanation for the gap detection deficits induced by sound exposure. First, the deficiency in gap detection at frequencies higher than the range used for tinnitus induction cannot easily be explained by either hearing loss or overall degraded performance. If hearing loss was an explanation, the major gap detection deficiency would have been expected within the range of sound exposure, not higher. Second, a complete recovery of ABR thresholds at the frequencies where gap detection deficits were persistent further supports tinnitus as a viable hypothesis. Third, sound exposure in our study was unilateral to ensure one fully functional ear for sufficient gap detection at control levels. Lastly, the vast majority of sound-exposed mice showed complete recovery of their prepulse detection performance to the control level. All these reasons make tinnitus, rather than hearing loss, the most plausible explanation for the gap detection deficits observed in sound exposed mice.

Our data suggest that $86 \%$ of sound-exposed mice developed behavioral signs of tinnitus, a percentage that is uncommonly high. Previous studies have shown that acoustic trauma can induce tinnitus symptoms in $56 \%$ (Kraus et al. 2010) to $75 \%$ (Wang et al. 2009) of exposed rats. There are three possible factors that may contribute to this inconsistency. First is that an overexposure caused by too loud sound may induce severe hearing loss. This is unlikely because the exposure delivered in the present study (1-h exposure to an octave band noise centered at $16 \mathrm{kHz}(116 \mathrm{~dB}$ SPL)) is considered a moderate exposure (McFadden et al. 1998; Fraenkel et al. 2003). Second, the differences in species between our study and the studies 
mentioned above may explain why a higher percentage of animals in the present study developed behavioral evidence of tinnitus following sound exposure. Third, the type of anesthesia employed can also be critical for determining the outcome of sound exposure. Kraus et al. (2010) reported that after a 2-h exposure to very loud sound (126 dB SPL), only 56\% of rats developed behavioral signs of tinnitus. Another study, which was also conducted on rats, reported that just $1 \mathrm{~h}$ of only $116 \mathrm{~dB}$ SPL sound exposure produced tinnitus symptoms in $75 \%$ of exposed rats (Wang et al. 2009). However, in the former study, sound exposure was performed under isoflurane anesthesia, whereas the latter used a mixture of ketamine and xylazine. It has been shown in mice that under isoflurane anesthesia, hearing loss caused by noise exposure is less severe than in non-anesthetized animals (Kim et al. 2005; Chung et al. 2007). Histological examination further revealed that hair cell survival was higher in the anesthetized mice. It is possible that under ketamine anesthesia, animals are more susceptible to the acoustic trauma. As ketamine was used in the present study, the sound exposure was putatively more effective for induction of tinnitus.

One seemingly contradictory phenomenon in the present study is the mismatch between recovery of startle and ABR amplitudes in the sound exposed mice. Even after 3 months of recovery following sound exposure, the amplitude of the startle does not return to pre-exposure levels (Fig. 7), whereas consistent with other studies (Bauer and Brozoski 2001; Turner et al. 2006; Wang et al. 2009), ABR showed complete recovery within this time period (Fig. 6). These data raise two fundamental questions: first, whether the peripheral auditory system completely recovers after a 1-h exposure to $116 \mathrm{~dB}$ SPL sound, and second whether ABR is a reliable measure of this recovery. Results of a recent study strongly suggest that the answer to both these questions is no (Kujawa and Liberman 2009). In this study, even a relatively low-level sound exposure (100 dB SPL) caused both an acute loss of afferent nerve terminals and a delayed degeneration of the cochlear nerve in $\mathrm{CBA} / \mathrm{CaJ}$ mice. At the same time, a moderate, but completely reversible, threshold shift was detected when ABRs were recorded. The authors concluded that ABR thresholds are sensitive metrics of hair cell damage but are insensitive to neuronal degeneration in the cochlea. Our observation of ABR recovery despite remaining behavioral evidence of tinnitus supports this idea. Although, the attenuation of startle response caused by sound exposure can have peripheral or/and central origins, we speculate that it may be used as a measure of neuronal degeneration in the cochlea. Future studies are necessary to clarify this issue.
Our prepulse data demonstrated that: (1) at day 1 post-exposure, a majority of mice showed little change in prepulse detection and several mice even showed detection improvement, (2) sound evoked changes in prepulse detection returned to the control level in less than 1 week and then were evident again between days 42 and 49 post-exposure. Such fast recovery of prepulse detection performance after exposure is not surprising because it has been described earlier in rats (Turner et al. 2006). Even the fact that some mice showed an improvement in prepulse detection right after sound exposure is not perplexing. This phenomenon has been related to hyperacusis developed in response to sound exposure (Bauer and Brozoski 2001; Turner et al. 2006). The most surprising aspect of our results is that after a complete recovery, the prepulse detection performance was reduced again between weeks 6 and 7 postexposure. Thus, recovery of the auditory system after sound exposure seems to involve multiple phases and requires further investigation.

In summary, the mouse tinnitus model has the capacity to detect and qualify symptoms of chronic tinnitus caused by sound exposure. This model also opens new avenues for research incorporating genetically modified animals. It is feasible to perform electrophysiological recordings in awake mice (Portfors and Roberts 2007; Portfors et al. 2009; Voytenko and Galazyuk 2011), allowing the combination of electrophysiological and psychoacoustic approaches to study the pathophysiology of tinnitus in a more comprehensive manner. Lastly, due to its robust hearing capabilities in comparison with other strains, the $\mathrm{CBA} / \mathrm{CaJ}$ strain of mice presents an opportunity for studying age effects in the development of tinnitus.

\section{ACKNOWLEDGMENTS}

We thank Merri Rosen and Jeremy Turner for their valuable comments on earlier versions of this manuscript. This work was supported by a Research Incentive grant from the Northeastern Ohio Universities College of Medicine.

\section{REFERENCES}

Ahmad N, Seidman M (2004) Tinnitus in the older adult: epidemiology, pathophysiology and treatment options. Drugs Aging 21:297-305

Bauer CA, Brozoski TJ (2001) Assessing tinnitus and prospective tinnitus therapeutics using a psychophysical animal model. J Assoc Res Otolaryngol 2:54-64

Bauer CA, Brozoski TJ, Rojas R, Boley J, Wyder M (1999) Behavioral model of chronic tinnitus in rats. Otolaryngol Head Neck Surg 121:457-462 
Brozoski TJ, Bauer CA, Caspary DM (2002) Elevated fusiform cell activity in the dorsal cochlear nucleus of chinchillas with psychophysical evidence of tinnitus. J Neurosci 22:2383-2390

Chung JW, Ahn JH, Kim JY, Lee HJ, Kang HH, Lee YK, Kim JU, Koo SW (2007) The effect of isoflurane, halothane and pentobarbital on noise-induced hearing loss in mice. Anesth Analg 104:1404 1408

Davis RR, Newlander JK, Ling X, Cortopassi GA, Krieg EF, Erway LC (2001) Genetic basis for susceptibility to noise-induced hearing loss in mice. Hear Res 155:82-90

Dong S, Mulders WH, Rodger J, Woo S, Robertson D (2010) Acoustic trauma evokes hyperactivity and changes in gene expression in guinea-pig auditory brainstem. Eur J Neurosci 31:1616-1628

Engineer ND, Riley JR, Seale JD, Vrana Wa, Shetake JA, Sudanagunta SP, Borland MS, Kilgard MP (2011) Reversing pathological neural activity using targeted plasticity. Nature 470:101-104

Erlandsson SI, Hallberg LR, Axelsson A (1992) Psychological and audiological correlates of perceived tinnitus severity. Audiology 31:168-179

Fraenkel R, Freeman S, Sohmer H (2003) Susceptibility of young adult and old rats to noise-induced hearing loss. Audiol Neurootol 8:129-139

GAO J, WU X, ZUo J (2004) Targeting hearing genes in mice. Brain Res Mol Brain Res 132:192-207

Guitton MJ, Caston J, Ruel JRM, Pujol R, Puel JL (2003) Salicylate induces tinnitus through activation of cochlear NMDA receptors. J Neurosci 23:3944-3952

Heffner HE, Harrington IA (2002) Tinnitus in hamsters following exposure to intense sound. Hear Res 170:83-95

Heffner HE, Koay G (2005) Tinnitus and hearing loss in hamsters (Mesocricetusauratus) exposed to loud sound. Behav Neurosci 119:734-742

Hirose K, Liberman MC (2003) Lateral wall histopathology and endocochlear potential in the noise-damaged mouse cochlea. J Assoc Res Otolaryngol 4:339-352

Ison JR, Castro C, Allen P, Virag TM, Walton JP (2002) The relative detectability for mice of gaps having different ramp durations at their onset and offset boundaries. J Accoust Soc Am 112:740 747

Kaltenbach JA, Afman CE (2000) Hyperactivity in the dorsal cochlear nucleus after intense sound exposure and its resemblance to tone-evoked activity: a physiological model for tinnitus. Hear Res 140:165-172

Kaltenbach JA, Zhang J, Afman CE (2000) Plasticity of spontaneous neural activity in the dorsal cochlear nucleus after intense sound exposure. Hear Res 147:282-292

Kim JU, Lee HJ, Kang HH, Shin JW, Ku SW, Ahn JH, Kim YJ, Chung JW (2005) Protective effect of isoflurane anesthesia on noiseinduced hearing loss in mice. Laryngoscope 115:1996-1999

Kraus KS, Mitra S, Jimenez Z, Hinduja S, Ding D, Jiang H, Gray L, Lobarinas E, Sun W, SAlvi RJ (2010) Noise trauma impairs neurogenesis in the rat hippocampus. Neurosci $167: 1216-1226$
KujaWa SG, Liberman MC (2009) Adding insult to injury: cochlear nerve degeneration after "temporary" noise-induced hearing loss. J Neurosci 29:14077-14085

Lobarinas E, Sun W, Cushing R, Salvi R (2004) A novel behavioral paradigm for assessing tinnitus using schedule-induced polydipsia avoidance conditioning (SIP-AC). Hear Res 190:109-114

McFadden SL, Campo P, Ding D, Quaranta N (1998) Effects of noise on inferior colliculus evoked potentials and cochlear anatomy in young and aged chinchillas. Hear Res 117:81-96

Mulders WH, Robertson D (2009) Hyperactivity in the auditory midbrain after acoustic trauma: dependence on cochlear activity. Neurosci 164:733-746

Mulders WH, Seluakumaran K, Robertson D (2010) Efferent pathways modulate hyperactivity in inferior colliculus. J Neurosci 30:9578-9587

PORTFORS CV, RoBERTS PD (2007) Temporal and frequency characteristics of cartwheel cells in the dorsal cochlear nucleus of the awake mouse. J Neurophysiol 98:744-756

Portfors CV, Roberts PD, Jonson K (2009) Over-representation of species-specific vocalizations in the awake mouse inferior colliculus. Neuroscience 162:486-500

Roberts Le, Eggermont JJ, Caspary DM, Shore SE, Melcher JR, Kaltenbach JA (2010) Ringing ears: the neuroscience of tinnitus. J Neurosci 30:14972-14979

RÜttiger L, Ciuffani J, Zenner HP, KNipPer M (2003) A behavioral paradigm to judge acute sodium salicylate-induced sound experience in rats: a new approach for an animal model on tinnitus. Hear Res 180:39-50

Salvi RJ, Wang J, Powers N et al (1996) Rapid functional reorganization in the inferior colliculus and cochlear nucleus after acute cochlear damage. In: Salvi RJ (ed) Auditory plasticity and regeneration. Thieme Medical Publishers, New York, pp 275-296

Turner JG (2007) Behavioral measures of tinnitus in laboratory animals. Prog Brain Res 166:147-156

Turner JG, Brozoski TJ, Bauer CA, Parrish JL, Myers K, Hughes LF, Caspary DM (2006) Gap detection deficits in rats with tinnitus: a potential novel screening tool. Behav Neurosci 120:188-195

VoYTEnKo SV, GALAZYUK AV (2011) mGluRs modulate neuronal firing in the auditory midbrain. Neurosci Lett 492:145-149

Wang H, Brozoski tJ, Turner JG, Ling L, Parrish JL, Hughes LF, CASPARY DM (2009) Plasticity at glycinergic synapses in dorsal cochlear nucleus of rats with behavioral evidence of tinnitus. Neurosci 164:747-759

Wu T, Marcus DC (2003) Age-related changes in cochlear endolymphatic potassium and potential in CD-1 and CBA/CaJ mice. J Assoc Res Otolaryngol 4:353-362

Yang G, Lobarinas E, Zhang L, Turner J, Stolzberg D, Salvi R, Sun W (2007) Salicylate induced tinnitus: behavioral measures and neural activity in auditory cortex of awake rats. Hear Res 226:244-253

Yoshida N, Hequembourg SJ, Atencio CA, Rosowski JJ, Liberman MC (2000) Acoustic injury in mice: $129 / \mathrm{SvEv}$ is exceptionally resistant to noise-induced hearing loss. Hear Res 141:97-106 